\title{
AN EXTENSION THEOREM FOR OBTAINING MEASURES ON UNCOUNTABLE PRODUCT SPACES
}

\author{
E. O. ELLIOTT
}

Abstract. Several theorems are known for extending consistent families of measures to an inverse limit or product space [1]. In this paper the notion of a consistent family of measures is generalized so that, as with general product measures [2], the spaces are not required to be of unit measure or even $\sigma$-finite. The general extension problem may be separated into two parts, from finite to countable product spaces and from countable to uncountable product spaces. The first of these is discussed in [3]. The present paper concentrates on the second. The ultimate virtual identity of sets is defined and used as a key part of the generalization and nilsets similar to those of general product measures [2] are introduced to assure the measurability of the fundamental covering family. To exemplify the extension process, it is applied to product measures to obtain a general product measure. The paper is presented in terms of outer measures and Carathéodory measurability; however, some of the implications in terms of measure algebras should be obvious.

Introduction. An uncountable product space $X=\prod_{i \in I} X_{i}$ is given with a family $D$ of countable subsets of the index set $I$. To each such subset $\tau \in \mathbb{D}$ there is an associated outer measure $\mu_{\tau}$ on the countable product space $X^{\tau}=\prod_{i \in \tau} X_{i}$. How the measures $\mu_{\tau}$ are obtained is not of interest here, but to keep the complete extension problem in mind we might think that $\mu_{\tau}$ is obtained as in [3] by extending a regular conditional measure system onto $X^{\tau}$. The problem we are concerned with here is that of stipulating conditions on the system of measures $\mu_{\tau}$ that allow their extension to an outer measure $\mu$ on $X$ having properties reflecting their own.

To proceed with the problem we need to agree on some notation. If $A \subset X$ and $\sigma$ is any subset of $I$, let $A_{\sigma}$ be the projection of $A$ onto the space $X^{\sigma}$, and if $a \subset X^{\sigma}$ let $a^{*}$ be the cylinder in $X$ over $a$. The symmetric difference between two sets $A$ and $B$ will be denoted by $A \triangle B$, and for $\tau \in \mathscr{D}$, its complement $I-\tau$ relative to $I$ will be denoted by $\tau^{\prime}$.

The family $D$ is said to be comprehensive when each countable subset $\sigma$ of $I$ is contained in some element $\tau$ of $D$ (i.e. $\sigma \subset \tau \in D)$ ). In the remainder of the paper we assume that $\mathscr{D}$ is comprehensive.

Received by the editors October 14, 1966 and, in revised form, May 5, 1967. 
1. The covering family. The existence of the covering family $\mathfrak{F}$ described below requires that the measures $\mu_{\tau}$ be rather congenially related. It is in this definition that the notion of a consistent family of measures is being generalized. To give the definition, we must first introduce two families of sets, the first of which is a traditional part of the extension process and the second of which is reminiscent of product measures. For $\tau \in \mathbb{D}$ let $\mathfrak{N}_{\tau}=\left\{\beta \subset X^{\tau}: \beta\right.$ is Carathéodory measurable with respect to $\left.\mu_{\tau}\right\}$ and

$$
\begin{aligned}
\AA_{\tau}= & \left\{\beta \subset X^{\tau^{\prime}}: \beta=\prod_{i \in \tau^{\prime}} \beta_{i} \text { where } \beta_{i} \subset X_{i} \text { for each } i \in \tau^{\prime},\right. \text { and } \\
& \left.(\alpha \times \beta)_{\sigma} \in \mathscr{N}_{\sigma} \text { for each } \alpha \in \mathscr{M}_{\tau} \text { and each } \sigma \in \mathcal{D} \text { such that } \tau \subset \sigma\right\} .
\end{aligned}
$$

For a covering family $\mathfrak{F}$ we require a family of subsets of $X$ such that for each $A \in \mathfrak{F}$ there exists $\tau \in \mathbb{D}$ with $A=\alpha \times \beta$ for some $\alpha \in \mathbb{M}_{\tau}$ and $\beta \in \mathbb{B}_{\tau}$, and $\mu_{\sigma}\left(A_{\sigma}\right)=\mu_{\tau}(\alpha)$ whenever $\sigma \in D$ and $\tau \subset \sigma$. (Note here, as a consequence of the definition of $\Theta_{\tau}$, that also $A_{\sigma} \in \mathfrak{M}_{\sigma}$.) Further restrictions will subsequently be placed on $\mathfrak{F}$.

In the above, $\beta$ is in some sense a set of unit measure relative to $\alpha$ and $A$ is the analogue of a classical cylinder set. On $\mathfrak{F}$ we can now define a function $\mu$ by means of $\mu(A)=\mu_{\tau}(\alpha)$ where $A=\alpha \times \beta, \alpha \in \mathscr{M}_{\tau}$, $\beta \in B_{\tau}, \tau \in D$, and $\mu_{\sigma}\left(A_{\sigma}\right)=\mu_{\tau}(\alpha)$ whenever $\sigma \in \mathbb{D}$ and $\tau \subset \sigma$. Using $\mu$ as a gauge and $\mathfrak{F}$ as a covering family, we generate an outer measure $\Psi$ on $X$ by taking $\Psi(A), A \subset X$, to be the infimum of numbers of the form $\sum_{B \in \mathcal{G}} \mu(B)$ where $\mathcal{G}$ is a countable subfamily of $\mathfrak{F}$ which covers $A$. We come now to our first

Theorem 1.1. If $A \in \mathfrak{F}$ then $\Psi(A)=\mu(A)$.

Proof. If $\mathcal{G}$ is a countable subfamily of $\mathfrak{F}$ such that $A \subset \cup \mathcal{G}$, then for each $B \in \mathcal{G} \cup\{A\}$ let $\tau_{B}$ be such a member of $D$ that $\mu(B)=\mu_{\tau_{B}}\left(B_{\tau_{B}}\right)$ and let $\sigma$ be such a member of $D$ that

$$
\bigcup_{B \in \mathcal{S} \cup\{A\}} \tau_{B} \subset \sigma .
$$

Thus $\mu(B)=\mu_{\sigma}\left(B_{\sigma}\right)$ for each $B \in \mathcal{G} \cup\{A\}$ and

$$
\mu(A)=\mu_{\sigma}\left(A_{\sigma}\right) \leqq \sum_{B \in \mathcal{S}} \mu_{\sigma}\left(B_{\sigma}\right)=\sum_{B \in \mathcal{S}} \mu(B),
$$

from which we may conclude that $\Psi(A)=\mu(A)$.

The first basic assumption to be made about $\mathfrak{F}$ is that if $\tau \in \mathscr{D}$, $\alpha \in \mathfrak{M}_{\tau}$, and $A \in \mathfrak{F}$, then $A \alpha^{*} \in \mathfrak{F}$ and $A-\alpha^{*} \in \mathfrak{F}$. This leads to our second theorem. 
Theorem 1.2. If $\tau \in \mathscr{D}$ and $\alpha \in \mathscr{T}_{\tau}$, then $\alpha^{*}$ is $\Psi$ measurable.

Proof. Since $\mathfrak{F}$ is the covering family for $\Psi$, it is sufficient to show that $\mu(T)=\mu\left(T \alpha^{*}\right)+\mu\left(T-\alpha^{*}\right)$ for each $T \in \mathfrak{F}$. Suppose then that $T \in \mathscr{F}$ and let $\sigma$ be such a member of $D$ that $\tau \subset \sigma$, each of the sets $\left(T \alpha^{*}\right)_{\sigma},\left(T-\alpha^{*}\right)_{\sigma}$ and $T_{\sigma}$ belong to $\mathfrak{M}_{\sigma}$ and $\mu\left(T \alpha^{*}\right)=\mu_{\sigma}\left(\left(T \alpha^{*}\right)_{\sigma}\right)$, $\mu\left(T-\alpha^{*}\right)=\mu_{\sigma}\left(\left(T-\alpha^{*}\right)_{\sigma}\right)$ and $\mu(T)=\mu_{\sigma}\left(T_{\sigma}\right)$. Then we have

$$
\mu(T)=\mu_{\sigma}\left(T_{\sigma}\right)=\mu_{\sigma}\left(\left(T \alpha^{*}\right)_{\sigma}\right)+\mu_{\sigma}\left(\left(T-\alpha^{*}\right)_{\sigma}\right)=\mu\left(T \alpha^{*}\right)+\mu\left(T-\alpha^{*}\right),
$$

which completes the proof.

The next basic assumption to be made about $\mathfrak{F}$ is that it be intersective, i.e. if $A \in \mathfrak{F}$ and $B \in \mathfrak{F}$, then $A B \in \mathfrak{F}$. With this we come to

2. Nilsets and ultimate virtual identity. Somewhat parallel to the definition of nilsets given in [2] we define a family of nilsets $\Re$ by

$\mathfrak{N}=\left\{N: N=\bigcup_{i \in I} n_{i}^{*}\right.$ where $n_{i} \subset X_{i}, A-N \in \mathfrak{F}$

$$
\text { and } \mu(A-N)=\mu(A) \text { whenever } A \in \mathfrak{F}\} \text {. }
$$

Two members $A$ and $B$ of $\mathfrak{F}$ are called ultimately virtually identical (u.v.i.) provided for some $\tau \in \mathscr{D}, \cup_{i \in \tau^{\prime}}\left(A_{\{i\}} \triangle B_{\{i\}}\right)^{*} \in \mathfrak{N}$. Since $D$ is comprehensive, $D$ is a directed set. The term ultimate refers to ultimate in the sense of the direction on $D$ and virtual identity refers to differences that amount to a nilset.

We now introduce a rather strong but natural assumption concerned with the idea that if two members of $\mathfrak{F}$ have much in common, then they are u.v.i. Specifically, our third assumption is that $\mathfrak{F}$ satisfies the condition that if $A \in \mathfrak{F}, B \in \mathfrak{F}$, and $\mu(A B)>0$ then $A$ and $B$ are u.v.i.

At this point we modify our measure $\Psi$ by requiring that members of $\Re$ have zero measure. We define $\phi$ to be the function on the subsets of $X$ such that

$$
\phi(A)=\inf _{N \in \mathscr{I}} \Psi(A-N)
$$

whenever $A \subset X$.

As our fourth and final assumption about our system of measures we ask that $\mathfrak{I}$ be closed to countable unions. Then $\phi$ turns out to be a measure which agrees with $\Psi$ on $\mathfrak{F}$ and may be generated by the covering family $\mathfrak{F} \cup \mathscr{Y}$ and a gauge $\mu^{\prime}$ which equals $\mu$ on $\mathfrak{F}$ but is zero on $\Re$. The point to the above modification of $\Psi$ is to achieve the measurability of the members of $\mathfrak{F}$. This brings us to 
Theorem 2.1. If $A \in \mathfrak{F}$, then $A$ is $\phi$ measurable and $\phi(A)=\Psi(A)$ $=\mu(A)$ and if $N \in \mathscr{N}$, then $\phi(N)=0$.

Proof. In view of the definitions of $\mathscr{N}$ and $\phi$ and Theorem 1.1 it is evident that $\phi(A)=\Psi(A)=\mu(A)$ and that $\phi(N)=0$. To see that $A$ is $\phi$ measurable it is only necessary to check that $\phi(T)=\phi(T A)$ $+\phi(T-A)$ for each $T \in \mathfrak{F} \cup \mathfrak{N}$, since $\mathfrak{F} \cup \mathfrak{N}$ is a covering family for $\phi$. The above equation is trivially satisfied when $T \in \Re$ so let us suppose that $T \in \mathfrak{F}$. Now if $\mu(A T)>0$, let $\sigma_{1}$ be a member of $D$ for which $N_{1}=\bigcup_{i \in \sigma_{1^{\prime}}}\left(T_{\{i\}} \triangle A_{\{i\}}\right) * \in \mathfrak{N}$. In view of the properties of $\mathfrak{F}$ discussed at the beginning of $\$ 1$ we can take $\sigma_{2} \in D$ large enough that $A=A_{\sigma_{2}} \times \beta$ where $A_{\sigma_{2}} \in \mathfrak{M}_{\sigma_{2}}$ and $\beta \in \wp_{\sigma_{2}}$. Now let $\tau$ be such a member of $D$ that $\sigma_{1} \subset \tau$ and $\sigma_{2} \subset \tau$, and let $B=\left(A_{\tau}\right)^{*}$. Then check that $A=B \cap \bigcap_{i \in \tau^{\prime}} A_{\{i\}}^{*}$ and with the aid of Theorem 1.2 infer that $B$ is $\Psi$ measurable and hence also $\phi$ measurable. Let $N=\bigcup_{i \in \tau^{\prime}}\left(T_{\{i\}} \triangle A_{\{i\}}\right)^{*}$ and note that $\tau^{\prime} \subset \sigma_{1}^{\prime}$ and hence $N \subset N_{1}$. In view of this and the fact that $\phi\left(N_{1}\right)=0$, we conclude that $\phi(N)=0$ also.

Now, since $A=B \cap \bigcap_{i \in \tau^{\prime}} A_{\{i\}}^{*}$, we have

$$
\begin{aligned}
T-A & =T-B \cup \bigcup_{i \in \tau^{\prime}}\left(T-A_{\{i\}}^{*}\right) \subset T-B \cup \bigcup_{i \in \tau^{\prime}}\left(T_{\{i\}}^{*}-A_{\{i\}}^{*}\right) \\
& =T-B \cup \bigcup_{i \in \tau^{\prime}}(T\{i\}-A\{i\})^{*} \subset T-B \cup \bigcup_{i \in \tau^{\prime}}(T\{i\} \triangle A\{i\})^{*}
\end{aligned}
$$

from which we infer that $T-A \subset T-B \cup N$. Noting further that $A \subset B$, we conclude

$$
\begin{aligned}
\phi(T) & \leqq \phi(T A)+\phi(T-A) \leqq \phi(T B)+\phi(T-B \cup N) \\
& \leqq \phi(T B)+\phi(T-B)+\phi(N)=\phi(T)+0
\end{aligned}
$$

and

$$
\phi(T)=\phi(T A)+\phi(T-A) .
$$

Now, if $\mu(A T)=0$, then

$$
\phi(T) \leqq \phi(T A)+\phi(T-A) \leqq 0+\phi(T-A) \leqq \phi(T) .
$$

Hence $\phi(T)=\phi(T A)+\phi(T-A)$ whenever $T \in F$ and the proof is complete.

3. An application to product measures. Suppose that for each $i \in I, \lambda_{i}$ is an arbitrary (outer) measure on $X_{i}$ and that $\mathscr{D}$ is the family of countable subsets of $I$. Then $D$ is clearly comprehensive. Now, for $\tau \in \mathfrak{D}, \tau=\left\{i_{1}, i_{2}, \cdots, i_{r}, \cdots\right\}$, we can define a regular conditional measure system $\nu_{\tau}$ on $X^{\tau}$ by taking $\nu_{0}=\lambda_{i_{1}}$ and $\nu_{r}(x, \cdot)=\lambda_{i_{r}+1}(\cdot)$ for 
each $x \in \prod_{t=1}^{r} X_{i_{i}}$. Then, by the construction in [3] we obtain from this regular conditional measure system a measure $\mu_{\tau}$ on $X^{\tau}$ for which

$$
\mu_{\tau}(\beta)=\prod_{r=1}^{\infty} \lambda_{i_{r}}\left(\beta_{r}\right)
$$

where $\beta=\prod_{r=1}^{\infty} \beta_{r}$ and for each $r, \beta_{r}$ is a $\lambda_{i_{r}}$ measurable subset of $X_{i_{r}}$ and $\prod_{r=1}^{\infty} \lambda_{i_{r}}\left(\beta_{r}\right)<\infty$.

For the system $\mu_{\tau}, \tau \in \mathcal{D}$, it can be shown that we can take

$\mathfrak{F}=\left\{A\right.$ : for some $\tau \in \mathscr{D}, A=\alpha \times \beta$ where $\alpha$ is a $\mu_{\tau}$ measurable set, $\mu_{\tau}(\alpha)<\infty$, and $\beta=\prod_{i \in \tau^{\prime}} \beta_{i}$ where for each $i \in \tau^{\prime}$, $\beta_{i}$ is a $\lambda_{i}$ measurable subset of $X_{i}$ and (1) $\mu_{\tau}(\alpha)=0$ or

and

(2) $\mu_{\tau}(\alpha)>0$ and $\lambda_{i}\left(\beta_{i}\right)=1$ for each $\left.i \in \tau^{\prime}\right\}$

$$
\mathfrak{x}=\left\{N: N=\bigcup_{i \in I} n_{i}^{*} \text { where } \lambda_{i}\left(n_{i}\right)=0 \text { for each } i \in I\right\} .
$$

It is clear that if $A \in \mathfrak{F}, B \in \mathfrak{F}$ and $\mu(A B)>0$ then for some $\tau \in \mathcal{D}$,

$$
\lambda_{i}\left((A B)_{(i\})}=1 \quad \text { for each } i \in \tau^{\prime}\right.
$$

and consequently $A$ and $B$ are u.v.i. Furthermore, $A B \in \mathfrak{F}$. If $\mu(A B)=0$, then for some $\sigma \in D, \mu_{\sigma}\left((A B)_{\sigma}\right)=0$ and again we see that $A B \in \mathfrak{F}$. Hence $\mathfrak{F}$ is intersective. Noting finally that $\mathfrak{T}$ is closed to countable unions we see that all of our assumptions are met and we obtain the measure $\phi$ on $X$ with the properties stated in Theorem 2.1. This measure is essentially the general product measure of [2]. By breaking the extension in to two parts, finite to countable, and countable to uncountable, the end result is reached more simply here than it is in [2].

\section{REFERENCES}

1. J. R. Choksi, Inverse limits of measure spaces, Proc. London Math. Soc. (3) 8 (1958), 321-342.

2. E. O. Elliott and A. P. Morse, General product measures, Trans. Amer. Math. Soc. (2) 110 (1964), 245-283.

3. E. O. Elliott, Measures on countable product spaces, Pacific J. Math. (to appear).

Bell Telephone laboratories, Inc., Holmdel, New Jersey 